\title{
Höhere zerebrale Quecksilberspiegel, aber geringere Alzheimer-Pathologie
}

Fragestellung: Gibt es einen Zusammenhang zwischen dem vermehrten Verzehr von Fisch und Meeresfrüchten und der zerebralen Quecksilberbelastung sowie der Alzheimer-Pathologie?

Hintergrund: Es gibt Hinweise auf neuroprotektive Effekte durch den Verzehr von Fisch und Meeresfrüchten (im Englischen unter "Seafood“ zusammengefasst), die der Entwicklung der Alzheimer-Pathologie (Amyloid-Plaques und Tau-Fibrillen) entgegenwirken. Diese Effekte werden insbesondere auf die vermehrte Konzentration von langkettigen N3-Fettsäuren und a-Linolensäure in Seafood zurückgeführt. Gleichzeitig bedingt ein vermehrter Konsum von Seafood eine erhöhte Quecksilberaufnahme, die potenziell neurotoxisch ist.

Patienten und Methodik: Bei einer Kohorte von Altenheimbewohnern $(\mathrm{n}=286)$ aus der Region um Chicago/Illinois (USA), die im Zeitraum von 2004 bis 2013 verstorben und die in Bezug auf ihre Ernährungsgewohnheiten prämortal befragt worden waren, wurden neuropathologische Untersuchungen des Gehirns durchgeführt. Als unabhängige Variable wurde aus den Informationen über die Häu-

Morris MC, Brockman J, Schneider JA et al. Association of seafood consumption, brain mercury level, and APOE ع4 status with brain neuropathology in older adults. JAMA 2016; 315: 489-97 figkeit des Konsums verschiedener Seafood-Produkte ein Schätzwert für die Einnahme von langkettigen N3-Fettsäuren und von a-Linolensäure berechnet. Es wurden die Quecksilber- und Selenkonzentrationen in zwei kortikalen Regionen (inferiorer Temporallappen und medialer Frontallappen) und im Kleinhirn bestimmt. Es erfolgte außerdem eine quantitative Bestimmung der für eine Alzheimer-Krankheit charakteristischen Veränderungen (AmyloidPlaques und Tau-Fibrillen) in multiplen Hirnregionen. In einem linearen Regressionsmodell mit den Ernährungsparametern (geschätzte Einnahme langkettiger N3-Fettsäuren und von a-Linolensäure) als prädiktive Variable und unter Kontrolle von Alter, Geschlecht und Bildungsgrad wurde deren Effekt auf die Konzentration der bestimmten Metalle und das Ausmaß der Alzheimer-Pathologie berechnet. Darüber hinaus wurden die Ergebnisse bei Trägern und Nichtträgern eines ApoE4-Allels (eines etablierten genetischen Risikofaktors für die AlzheimerKrankheit) getrennt analysiert.

Ergebnisse: Der Konsum von Fisch und Meeresfrüchten war einerseits mit einer erhöhten zerebralen Quecksilberkonzentration assoziiert. Andererseits gab es einen negativen Zusammenhang zwischen Seafood-Konsum und Alzheimer-Pathologie, der allerdings nur bei Trägern eines ApoE4-Allels signifikant war.

Schlussfolgerungen: Trotz einer verstärkten Aufnahme von Quecksilber hat ein erhöhter Konsum von Fisch und Meeresfrüchten möglicherweise einen protektiven Effekt im Hinblick auf die Entwicklung der Alzheimer-Pathologie, insbesondere bei Menschen mit einem genetisch erhöhten Risiko, die Alzheimer-Krankheit zu entwickeln.

\section{- Kommentar von Klaus Fließbach, Bonn}

\section{Schützt regelmäßiger Seafood-Konsum vor Alzheimer?}

Korrelative, querschnittliche Analysen sind naturgemäß in ihrer Aussagekraft begrenzt. Der in dieser Studie gezeigte negative Zusammenhang von Seafood-Konsum und Alzheimer-Pathologie kann viele Ursachen haben (z. B. die Vermeidung anderer, potenziell schädlicher Substanzen in anderen Nahrungsmitteln), die grundsätzlich nicht vollständig kontrollierbar sind. Dennoch stellt diese methodisch sehr saubere Arbeit einen Hinweis auf mögliche protektive Effekte von langkettigen N3-Fettsäuren und von a-Linolensäure für die Alzheimer-Krankheit dar, dem weiter nachgegangen werden sollte. Der Befund, dass gleichzeitig höhere zerebrale Quecksilberkonzentrationen bei Personen mit hohem SeafoodKonsum gemessen wurden, legt nahe, dass dies in Bezug auf die Alzheimer-Pathologie keinen wesentlichen, negativen Effekt hat.

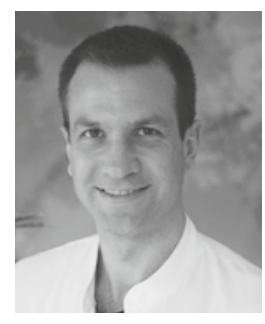

PD Dr. med. Dipl.-Psych. Klaus Fließbach, Bonn

Leiter des Klinischen Behandlungs- und Forschungszentrums für neurodegenerative Erkrankungen (KBFZ)

E-Mail: klaus.fliessbach@ukb.uni-bonn.de 